\title{
High-power laser shock-induced dynamic fracture of aluminum and microscopic observation of samples
}

\author{
Zhang Fan ${ }^{1}$, Huang Xiuguang ${ }^{1, a}$, Shu Hua ${ }^{1}$, Xiao Dawu², He Lifeng ${ }^{2}$, Xie Zhiyong ${ }^{1}$, Ye Junjian ${ }^{1}$, Dong Jiaqin ${ }^{1}$, Jia guo ${ }^{1}$, \\ Fang Zhiheng ${ }^{1}$, and Zhou Huazhen ${ }^{1}$ \\ ${ }^{1}$ Shanghai Institute of Laser Plasma(SILP), ShangHai 201800, China \\ ${ }^{2}$ Materials Research Institute, China Academy of Engineering Physics, Mian Yang 621900, China
}

\begin{abstract}
High-power laser induced shocks generated by "ShenGuang II" laser facility has been used to study spall fracture of polycrystalline aluminum at strain rates more than 106/s. The free surface velocity histories of shock-loaded samples, $150 \mu \mathrm{m}$ thick and with initial temperature from $293 \mathrm{~K}$ to $873 \mathrm{~K}$, have been recorded using velocity interferometer system for any reflector (VISAR). From the free surface velocity profile, spall strength and yield stress are calculated, it demonstrates that spall strength will decline and yield strength increase with initial temperature. The loaded samples are recovered to obtain samples' section and free surface metallographic pictures through Laser Scanning Confocal Microscopy. It is found that there are more microvoids and more opportunity to appear bigger voids near the spall plane and the grain size increases with temperature slowly but smoothly except the sharply change at $893 \mathrm{~K}$ (near melting point). Besides, the fracture mechanisms change from mainly intergranular fracture to transgranular fracture with the increase of initial temperature.
\end{abstract}

\section{Introduction}

The research of materials dynamic damage characteristics has great significance in many fields such as high speed collision of automobile, aerospace, new material synthesis, satellite protection, weapons design etc. Spall is a typical dynamic fracture that takes place inside a solid body as a result of tensile stresses that develop due to the interaction of rarefaction waves. Brittle fracture occurs through the generation and propagation of micro cracks while ductile fracture proceeds through the nucleation, growth, and coalescence of micro voids [1]. In 1914, Hopkinson firstly observed the spall phenomenon [2] that there were planar separation of material parallel to the wave front when materials under dynamic loads. After the Second World War, driven by engineering and military applications, spall research gets widespread concern and has a great progress [3-5]. Since the 1970s, the concepts of damage accumulation and evolution have been proposed as being responsible for the dynamic fracture of ductile metals. Many scientists have endeavored to develop a theoretical equation for damage evolution on the basis of statistical analysis of damage of recovered samples, and more detailed information based on experimental observation is needed in order for one to better understand or modify these theoretical results. Different methods of dynamic loading have been tried to find materials' damage characteristics, mainly of these studies use SHPB(split Hopkinson pressure bar) with $10^{4} \mathrm{~s}^{-1}$ strain rates, and gas gun and explosives with strain rates up to $10^{4} \mathrm{~s}^{-1}$ $10^{6} \mathrm{~s}^{-1}$. Recent years, high-power lasers also have been used to generate spall in materials at much higher strain rate, sometimes up to $108 \mathrm{~s}^{-1}$, the laser is focused onto

$\overline{{ }^{a} \text { Corresponding author: innocentman } 0010163 . c 0 m}$ and absorbed at the front surface of the target, heating, and ablating the surface. The backwards-expelled material acts as a rocket engine pushing the front surface with a pressure strong enough to launch a shock wave into the material. This paper attempts to study aluminum dynamic damage characteristics through spall experiments at "ShenGuang II" laser facility. Meanwhile, microscopic observations of recovered samples have been done to get damage information and verify the results of macroscopic measurements such as spall strength and yield strength.

\section{Experimental technique}

The present study was performed using the "ShenguangII" Nd: Glass laser (converted to $\lambda=0.351 \mu \mathrm{m}$ ) of the National Laboratory on High Power Laser and Physics. The"Shenguang-II" laser facility provides onedimensional compression by smoothed laser beams with short wavelength and high intensity. The temporal profile of the laser is nearly square with a rise and fall time of $\sim 300$ ps and a full width at half maximum (FWHM) of $\sim 3$ ns. Lens-array (LA) [6-8] was used to eliminate the large scale spatial modulation and to obtain a flat-topped profile in the focal plane. Characteristics of the optical system (Lens+LA) were such that the focal spot had a $\sim 1 \mathrm{~mm}$ diameter flat region. The absorbed laser intensity in the focal spot was $\sim 10^{11} \mathrm{~W} / \mathrm{cm}^{2}$.

Foils of polycrystalline aluminum of $99.99 \%$ purity with $150 \mu \mathrm{m}$ thickness and different initial temperature were used in the experiment. Samples were measured to be fully dense $\left(2.71 \mathrm{~g} / \mathrm{cm}^{3}\right)$ to within an accuracy of $1 \%$. The longitudinal sound velocity $\mathrm{c}_{l}=6450 \pm 30 \mathrm{~m} / \mathrm{s}$ and the bulk sound velocity $\mathrm{c}_{b}=5360 \pm 30 \mathrm{~m} / \mathrm{s}$ were measured using a dynamic experiment. The metallography examination of the foils shows that the foils contain 


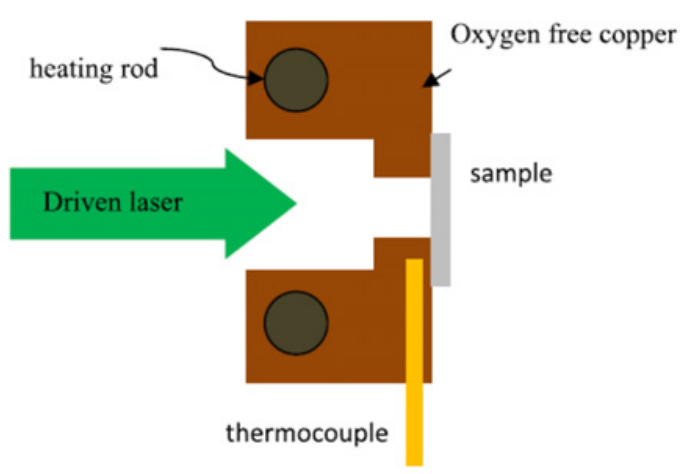

Figure 1. Schematic of the target.

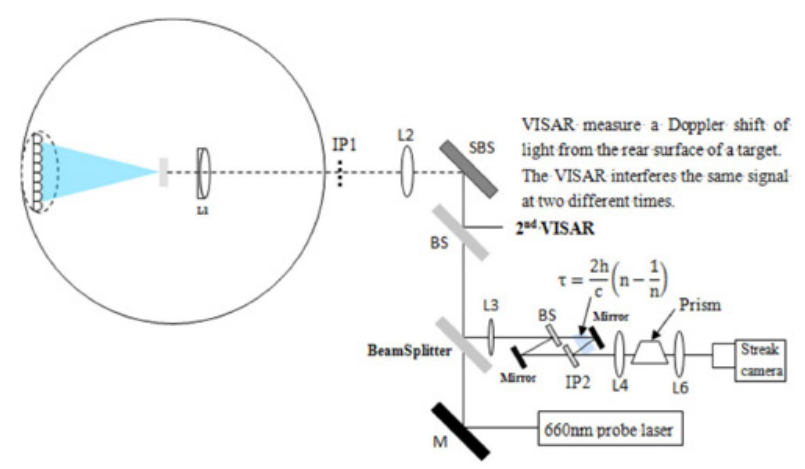

Figure 2. Schematic of the VISAR. L1-L6:Image Lens; IP1IP2:Image Position; LA: Lens Array; SBS: Special Beam Splitter.

globular grains, whose average diameter is approximately $20 \mu \mathrm{m}$. The shock-loaded samples were $10 \mathrm{~mm} \times 10 \mathrm{~mm}$ squares of different thicknesses. Each sample was glued to an oxygen-free copper target holder. The target holder had two heating rods. The heater power was sufficient to reach the melting temperature of aluminum after 15 min of heating. The temperature was controlled by a thermocouple placed near the sample. The experimental setup is shown in Fig. 1. The heating system could heat up the sample to $973 \mathrm{~K}$ with temperature controlling accuracy of $2 \mathrm{~K}$.

The experiment diagnostic system is a line VISAR [9, 10]. The schematic optical setup of the system is shown in Fig. 2. The total magnification of the image system is $11 \times$. The minimum scale of the resolution pattern is $7 \mu \mathrm{m}$ and the time resolution of the VISAR system was $\sim 100 \mathrm{ps}$ in this experiment.

After laser loading, recover the samples from target frame. Then obtain the metallographic samples through sampling, mosaic, grinding, polishing and etching. Finally make metallographic analysis using fracture surface and section pictures got by LSCM(laser scanning confocal microscope).

\section{Experiment results and discussion}

\subsection{Measurements of spall strength and yield stress}

Typical VISAR image of the experiment is shown in Fig. 3. The horizontal direction of the image is the space. The

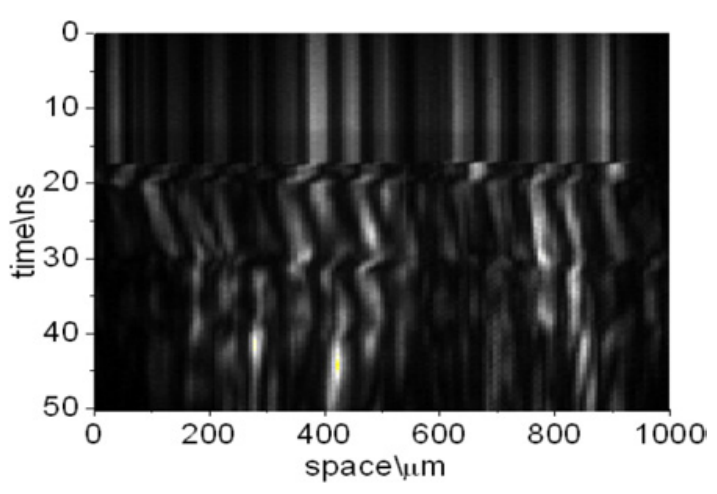

Figure 3. VISAR image of $873 \mathrm{~K}$ Aluminum sample.

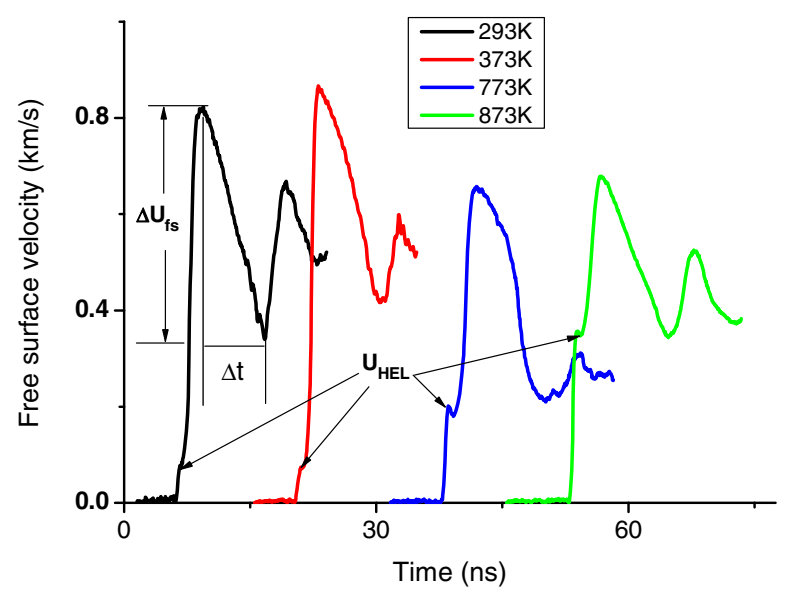

Figure 4. Free surface velocity profile of different initial temperature samples.

Table 1. Parameters of free surface velocity profile.

\begin{tabular}{ccccc}
\hline $\begin{array}{c}\text { Initial } \\
\text { Temperature } \\
(\mathrm{K})\end{array}$ & $\begin{array}{c}\text { Strain } \\
\text { Rate } \\
\left(10^{6} / \mathrm{s}\right)\end{array}$ & $\begin{array}{c}\text { Spall } \\
\text { strength } \\
\sigma_{s \mathrm{p}}(\mathrm{GPa})\end{array}$ & $\begin{array}{c}\text { HEL } \\
\text { strength } \\
\sigma_{\text {hel }}(\mathrm{GPa})\end{array}$ & $\begin{array}{c}\text { Yield } \\
\text { Strength } \\
\text { Y }(\mathrm{GPa})\end{array}$ \\
\hline 293 & 480 & 78 & 7.59 & 4.85 \\
373 & 450 & 73 & 7.25 & 4.85 \\
773 & 450 & 200 & 8.25 & 4.16 \\
873 & 340 & 360 & 8.12 & 3.16 \\
\hline
\end{tabular}

vertical direction is the time. The temporal scale of the image is $50 \mathrm{~ns}$.

According to the measurement principle of VISAR, we can get the free surface velocity profile of different temperature initial spall samples shown in Fig. 4.

From the free surface velocity profile, we can get information like HEL (Hugoniot Elastic Limit) velocity, pullback velocity and so on listed in the Table 1 .

Using the acoustic approximation formula (1), (2), (3), spall strength and yield stress of different initial temperature aluminum samples can be calculated through the HEL velocity and pullback velocity. The results are shown in Fig. 5.

$$
\sigma_{H E L}=\frac{1}{2} \rho_{0} c_{l} u_{H E L}
$$

$$
\mathrm{Y}=\frac{3}{2} \sigma_{H E L}\left(1-\frac{c_{b}^{2}}{c_{l}^{2}}\right)=\frac{3}{4} \rho_{0}\left(c_{l}-\frac{c_{b}^{2}}{c_{l}}\right) \mu_{H E L}
$$




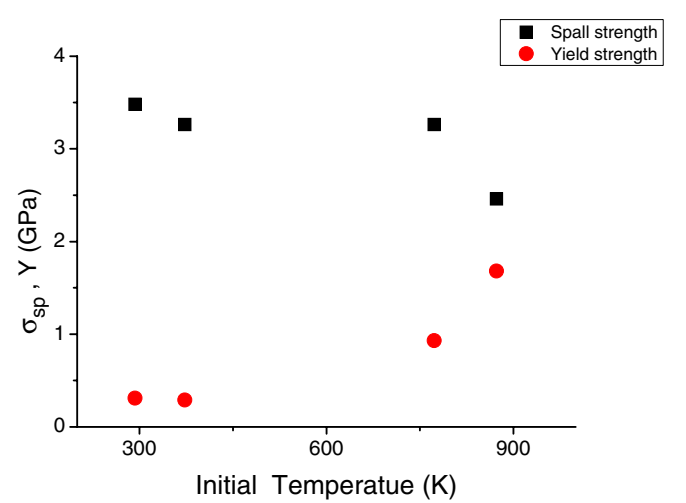

Figure 5. Yield stress and spall strength as a function of the initial temperature.

$$
\sigma_{s p}=\frac{1}{2} \rho_{0} c_{b} u_{f s} .
$$

We can find that the amplitude of elastic precursor increased with the increasing of the temperature and the spall strength decreased with temperature.

At low strain rates, overcoming obstacles by dislocations is due to the joint action of applied stress and thermal fluctuations. The increase of the fluctuations with temperature facilitates the motion of dislocations and leads to the decrease of the yield stress. At sufficiently high strain rates, phonon friction is the dominant mechanism that controls dislocation motion. The phonon-related dislocation drag is proportional to the temperature, so there is an increase of the yield stress with temperature. pure cobalt, silver, titanium, and some other crystalline solids also have similar characteristics [11-13].

On the other hand, Due to the theoretical models, the incipient stage of a high-rate spall fracture is the nucleation and growth of numerous voids or cracks. The resistance to growth of voids is dominated by the flow stress of the material around the void. So, the decrease of the yield strength with heating should lead to a decrease of spall strength. Since the spall strength data of Fig. 5 show a reverse trend. we can conclude that the spall strength of preheated aluminum is controlled more by the rate of void nucleation rather than its growth [14].

\subsection{Metallographic observation and analysis}

The recovered samples are first used to take pictures of target surface like Fig. 6. It is obvious that the front surface has a characteristic of melting and solidification reveal that the target has been ablated by high-power laser. The back surface (or free surface) has convex character, and incomplete spall samples have relatively complete morphology while complete spall samples have pits cause a part of materials parallel to surface have flown out.

Cut the samples along the impact direction symmetrically, take one piece and make cold mosaic with epoxy resin. Then, set the sample of epoxy resin column in the polishing machine fixture, using different particle size of sand paper step by step from coarse to fine polishing. When the surface polishing scratches is very small, polish with diamond polishing liquid; Finally, reveal
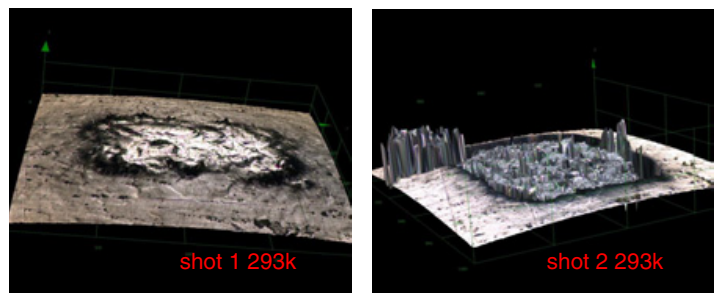

Figure 6. The back surface of incomplete and complete spall samples.

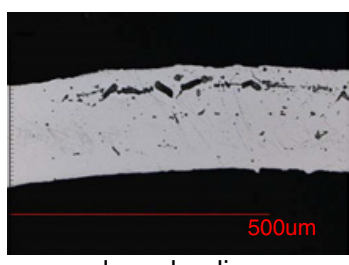

laser loading

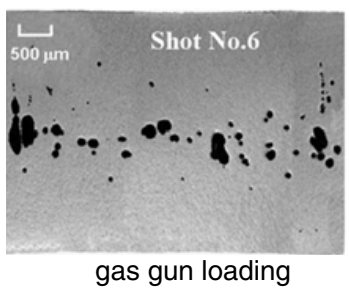

gas gun loading
Figure 7. Recovered samples of laser loading and gas gun loading.

metallographic structure by the erosion corrosion liquid, and further metallographic analysis can be done.

\subsubsection{Influence of strain rate on material strength}

Figure 7 is the metallographic section pictures of laser loading and gas gun driven flyer impact samples [15] respectively.

It is obvious that the voids are smaller and more irregular in samples of high-power laser loading than the gas gun loading, demonstrate that the growth time of voids of laser loading is much shorter which is closely related to strain rate, the strain rate of high-power laser loading is higher than that of gas gun loading. Considering the dynamic fracture of material damage cumulative time effect, to achieve the same material damage threshold, the laser loading spall need greater tensile stress than gas gun loading because of its shorter duration. Therefore under the same initial temperature, materials loading by high-power laser should have higher yield stress and spall strength, and this have been proved by lots of researches. Figure 8 is the comparison of this work and E. B. Zaretsky's work [16] which agree our prediction well.

\subsubsection{Effect of temperature on grain size}

The grain size of the sample is an important factor that affect the strength of materials properties, so we observed the grain size of the sample with the temperature changes, as shown in Fig. 9. It can be found that with the rise of temperature, grain size increases slowly and smoothly, but dramatically when close to the melting point. P. B. Trivedi et al. [17] found that with the increase of grain size, the proportion of grain boundary in materials decreases, and the nucleation point in high purity metal is mainly on the grain boundary, therefore with reduced nucleation point number, spall strength increases. In our work, with the increase of grain size, the spall strength decreases. Shows that other factors effect such as flow stress caused by temperature exceeds the grain size effect on the strength 

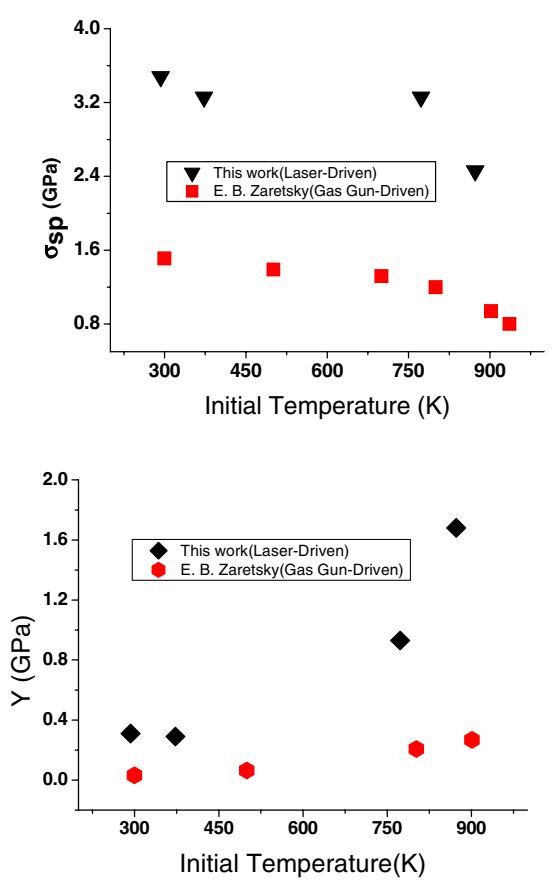

Figure 8. Spall strength and yield stress comparison of different loading.

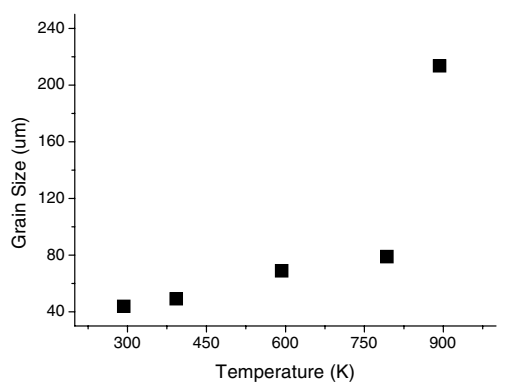

Figure 9. Grain size as a function of initial temperature.

of materials, shows that material damage characteristic is a combination of many kinds of factors, it also provides a useful reference information for damage evolution model.

\subsubsection{Effect of temperature on the material fracture mode}

Strain rate effects on material failure has been studied a lot $[18,19]$. With the increase of strain rate, the dynamic fracture mode will change from the intergranular fracture primarily to transgranular fracture. Dynamic fracture is a time related to evolution process, time effect or rate effect mainly influence the grow process of the microvoids; However when the strain rate is in the same order of magnitude, the influence of thermal effects on the material fracture mode needs more consideration. Figure 10 is section picture of samples with the initial temperature of $373 \mathrm{~K}, 573 \mathrm{~K}$ and $873 \mathrm{~K}$ respectively. It can be seen that the material fracture mode changes from mainly the intergranular fracture (shot 3 and shot 4) to mainly the transgranular fracture (shot 7). With the increase of temperature, "fluid" properties of the material appear more, and the difference of damage threshold of grain boundary and inside grain will be smaller. On

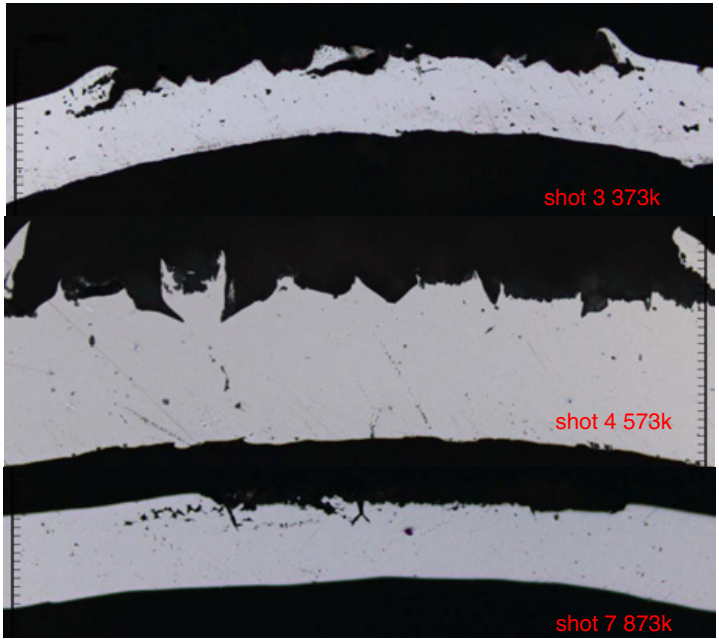

Figure 10. Effect of initial temperature on the material fracture mode.

the other hand, thermal fluctuation becomes the main factor of nucleation in high purity metal, making the void distribution more dense and the distance of the adjacent voids shorter, so the coalescence of void in different position like inside grain and on grain boundary will be much easier as temperature increasing. Therefore, the material will show the transgranular fracture mode more. It is important to note that the original sample thickness is about $150 \mu \mathrm{m}$, and in $873 \mathrm{~K}$ material's grain size is about $200 \mu \mathrm{m}$, thus the change of fracture mode may be a scale effect. whether thicker samples have the same nature need follow-up experiments to verify.

\section{Conclusion}

Spall experiments are conducted on "ShenGuang II" laser facility to study the effect of initial temperature on aluminum at a approximately same strain rate with same thickness samples. It can be concluded that with the increase of initial temperature, spall strength decrease and the yield stress increases; Material grain size increases with temperature slowly, but wheb near the melting point it increases dramatically; Materials fracture mode changes from mainly the intergranular fracture to mainly the transgranular fracture with the increase of initial temperature.

\section{References}

[1] T. Antoun, L. Seaman, D. Curran, G. Kanel, S. Razorenov, A. Utkin, Spall Fracture, p 3-4 (2002)

[2] HoPkinson, B. Philosophical Transactions of the Royal Society of London, 213(A), 437 (1914)

[3] Lee Davison, A. L. Stevens, J. Appl. Phys. 43(3), 988 (1972)

[4] Lynn Seaman, Donald R. Curran, Donald A. Shockey, J. Appl. Phys. 47(11), 4814 (1976)

[5] Grady, D.E. J. Mech. Phys. Solids 36(3), 353 (1988)

[6] Deng Ximing, Liang Xiangchun, Chen Zunze, et al., Chinese Journal of Lasers 12(5), 257 (1984)

[7] Fu Sizu, Sun Yuqin, Huang Xiuguang, et al., Chinese Journal of Lasers 30(2), 129 (2003) 
[8] Huang Xiuguang, Fu Sizu, Wu Jiang, et al., High Power Laser and Particle Beams 18(5), 811 (2006)

[9] Shu Hua, Fu Sizu, Huang Xiuguang, et al., Chinese Journal of Lasers 37(1), 176 (2010)

[10] Shu Hua, Fu Sizu, Huang Xiuguang, et al., Acta physica sinica 61(11), 114102 (2012)

[11] G.V. Garkushin, G.I. Kanel, S.V. Razorenov, Phys. Solid State. 52(11), 2369 (2010)

[12] E.B. Zaretsky, J. Appl. Phys. 108, 083525(2010)

[13] E.B. Zaretsky, G.I. Kanel, J. Appl. Phys. 110, 073502 (2011)
[14] Hua Shu, Sizu Fu, Xiuguang Huang et al., J. Appl. Phys. 116, 033506 (2014)

[15] Meilan Qi, Chao Luo, Hongliang He, et al., J. Appl. Phys. 111, 043506 (2012)

[16] E.B. Zaretsky, G.I. Kanel, J. Appl. Phys. 112, 073504 (2012)

[17] P.B. Trivedi, J.R. Asay, Y.M. Gupta, et al., J. Appl. Phys. 102, 083513 (2007)

[18] E. Moshe, S. Eliezer, E. Dekel, A. Ludmirsky, et al., J. Appl. Phys. 83(8), 4004 (1998)

[19] S.V. Razorenov, G.I. Kanel, K. Baumung, et al., AIP Conference Proceedings, 620, 503 (2002) 